\title{
DRAG AND PRESSURE FIELDS FOR THE MHD FLOW AROUND A CIRCULAR CYLINDER AT INTERMEDIATE REYNOLDS NUMBERS
}

\author{
T. V. S. SEKHAR, R. SIVAKUMAR, AND T. V. R. RAVI KUMAR
}

Received 26 November 2004 and in revised form 11 April 2005

Steady incompressible flow around a circular cylinder in an external magnetic field that is aligned with fluid flow direction is studied for Re (Reynolds number) up to 40 and the interaction parameter in the range $0 \leq N \leq 15$ ( or $0 \leq M \leq 30$ ), where $M$ is the Hartmann number related to $N$ by the relation $M=\sqrt{2 N \text { Re }}$, using finite difference method. The pressure-Poisson equation is solved to find pressure fields in the flow region. The multigrid method with defect correction technique is used to achieve the second-order accurate solution of complete nonlinear Navier-Stokes equations. It is found that the boundary layer separation at rear stagnation point for $\mathrm{Re}=10$ is suppressed completely when $N<1$ and it started growing again when $N \geq 9$. For $\operatorname{Re}=20$ and 40 , the suppression is not complete and in addition to that the rear separation bubble started increasing when $N \geq 3$. The drag coefficient decreases for low values of $N(<0.1)$ and then increases with increase of $N$. The pressure drag coefficient, total drag coefficient, and pressure at rear stagnation point vary with $\sqrt{N}$. It is also found that the upstream and downstream pressures on the surface of the cylinder increase for low values of $N(<0.1)$ and rear pressure inversion occurs with further increase of $N$. These results are in agreement with experimental findings.

\section{Introduction}

The analytical calculations of the magnetohydrodynamic flow around an obstacle encounters difficulties due to the boundary layer separation occurring at high Reynolds numbers in downstream zone. For the particular case of a cylindrical obstacle with a diameter $d$, immersed in a liquid metal flow which has a uniform velocity $U_{\infty}$ at infinity and aligned with the magnetic field $B_{0}$, Tamada [21] gives a general solution for the vorticity distribution throughout an inviscid fluid. For the case of very low interaction parameter $N$ (i.e., $N \ll 1$ ), where the magnetic field effect upon the flow is weak (interaction parameter signifies the ratio of the electromagnetic to the inertial forces), Tamada applied a perturbation technique to the nonmagnetic flow and found that the first-order term of the induced vorticity shows a discontinuity in the downstream region. For the same range of small values of the nondimensional parameters $N$ and $R_{m}\left(R_{m}\right.$, the magnetic Reynolds 
number, is given by $U_{\infty} a \mu \sigma$ ), Lahjomri [10] has clearly depicted the flow around a cylindrical obstacle for this situation, where the flow and magnetic field are aligned at infinity. Using the same Oseen-type perturbation technique, but taking the magnetic field perturbation into account, he found a vorticity field which is in complete agreement with that of Tamada at large distances from the cylinder. Lahjomri shows a decrease of the upstream tangential velocity at the cylinder surface when compared to the potential flow solution. In contrast, the downstream flow is accelerated by the electromagnetic force reducing the adverse pressure gradient and the tendency of the boundary layer to separate. The magnetohydrodynamic flow around a circular cylinder for low $\operatorname{Re}(<10)$ is studied by Bramely $[3,4]$ using Oseen approximation and the method of series truncation. $\mathrm{He}$ found that for very low values of the interaction parameter $N$, the drag coefficient decreases and then increases with increase of $N$. Swarup and Sinha [19] investigated the steady flow of an incompressible, viscous, electrically conducting fluid past a nonmagnetic and nonconducting circular cylinder for low Reynolds numbers and low Hartmann numbers $M$ using the method of matched asymptotic expansion. (The Hartmann number $M$ is defined as $\left.\mu H_{\infty} a(\sigma / \eta)^{1 / 2}\right)$.

It is known that the flow around a cylinder becomes unstable at $\operatorname{Re} \approx 49$ due to the periodic vortex shedding. For the cylinder flow, Lahjomri et al. [11] experimentally verified that the 2D instability (vortex shedding) can be suppressed by applying an aligned magnetic field. The conditions needed to facilitate the suppression of von Kármán vortex excitation of a circular cylinder by a second cylinder set downstream in a cruciform arrangement is investigated by Bae et al. [1]. Recently, Baranyi [2] studied the unsteady momentum and heat transfer from a fixed cylinder in an aligned magnetic field. It has been shown by Mutschke et al. [15] that the 3D steady flow does exist. Later Mutschke et al. [16] found the 3D instabilities of the cylinder wake in an external magnetic field. In our previous paper [18], we have studied the hydrodynamic flow of a conducting fluid around a sphere in an aligned magnetic field for moderate values of interaction parameter and observed the nonmonotonic behavior of the separation angle and separation length. In the experimental studies on sphere, Maxworthy [13, 14] and Yonas [23] discussed the pressure distributions in both upstream and downstream zones and the asymptotic dependence with $N$ of the drag coefficient. Josserand et al. [8] presented pressure distribution measurements around a cylinder placed in a liquid metal flow aligned with a constant magnetic field. He found that the pressure is reduced by the electromagnetic forces for values of the interaction parameter $N<1$. For higher values of $N$, the rear pressure drag and the global pressure drag exhibit a $\sqrt{N}$ dependence and it is also shown that for a sufficient value of the magnetic field, the von Kármán street behind the cylinder can be suppressed. In the absence of the magnetic field, the results are given by Takami and Keller [20], Dennis and Chang [5], Fornberg [6, 7], and Silva et al. [12].

If a fluid is electrically conductive, its flow may be controlled using electromagnetic forces. Meanwhile, this technique is a recognized tool even on an industrial scale for handling highly conductive materials like liquid metals and semiconducting melts. However, fluids of low electrical conductivity like sea water also permit electromagnetic flow control. Because separation of flow is considered an undesirable feature, a considerable amount of research has been devoted to the control of flow separation. Besides the control 
by electromagnetic forces, there are techniques like suction, blowing, and wall movement for the control of separation. A renewed interest in the use of electromagnetic forces to control the flow of electrolytes arose in the 1990s.

In this paper, we discuss the nature of incompressible conducting fluid flow around a circular cylinder in the presence of an external magnetic field for a range of Reynolds numbers from 10-40 and with interaction parameter $0 \leq N \leq 15$ (or $0 \leq M \leq 30$ ), using the finite difference method. The multigrid method with defect correction technique is applied to obtain the second-order accurate solution. It is found that the boundary layer separation length and separation angle behaves in a nonmonotonic way with an increase in magnetic field. The behavior of drag coefficient and pressure are found to agree with the experimental findings. The paper is organized as follows. In Section 2, we define the MHD flow problem and discuss the governing equations. In Section 3, we briefly describe the applied numerical method. Section 4 discusses our main results on separation point, separation length, drag coefficient, and pressure. In the conclusion we give a short summary and an outlook towards further future research.

\section{Formulation of the problem}

The equations governing the steady MHD flow of an incompressible fluid (with finite electrical conductivity $\sigma$ ), past a circular cylinder (of radius $a$ ), with uniform free-stream velocity $\mathbf{U}_{\infty}$ and uniformly applied magnetic field $\mathbf{H}_{\infty}$, at large distances in nondimensional form, is as follows.

(i)Curl of momentum equation:

$$
\nabla^{2} \boldsymbol{\omega}=\frac{\operatorname{Re}}{2}[\nabla \times(\boldsymbol{\omega} \times \mathbf{q})]-\frac{N \operatorname{Re}}{2}[\nabla \times\{(\mathbf{q} \times \mathbf{H}) \times \mathbf{H}\}]
$$

in which

$$
\boldsymbol{\omega}=\nabla \times \mathbf{q}
$$

(ii) Equation of continuity:

$$
\nabla \cdot \mathbf{q}=0
$$

$\mathbf{q}$ is the fluid velocity, $\mathbf{H}$ the magnetic field, and $\omega$ the vorticity. The Reynolds number Re is given by $\operatorname{Re}=2 \rho U_{\infty} a / \eta$ and $N=\sigma H_{\infty}^{2} a / \rho U_{\infty}$ is the interaction parameter. The viscosity and density of the fluid are $\eta$ and $\rho$, respectively. In order to satisfy (2.3), the dimensionless stream function $\psi(r, \theta)$ is introduced so that

$$
u=\frac{1}{r} \frac{\partial \psi}{\partial \theta}, \quad v=-\frac{\partial \psi}{\partial r}
$$

where $u$ and $v$ are the dimensionless radial and transverse components of fluid velocity. Cylindrical polar coordinates $(r, \theta, z)$ are used in such a way that the flow is symmetric about $\theta=0^{\circ}$ and $\theta=180^{\circ}$. As the magnetic field and fluid flow are aligned at infinity, the electric field can be assumed to be zero. The problem can be simplified by assuming the 
magnetic Reynolds number $R_{m}$ (ratio of the induced magnetic field to imposed magnetic field) to be small. We use the low $-R_{m}$ approximation and replace the magnetic field in all MHD equations by

$$
\mathbf{H}=(-\cos \theta, \sin \theta, 0)
$$

which will eliminate several nonlinear terms of unknown quantities in the governing equations. Substitution of (2.4) in (2.2) and (2.4), (2.5) in (2.1) with the transformation $r=e^{\pi \xi}$ and $\theta=\pi \eta$ yields, in the vorticity-stream function form, the following:

$$
\begin{gathered}
\frac{\partial^{2} \psi}{\partial \xi^{2}}+\frac{\partial^{2} \psi}{\partial \eta^{2}}+\pi^{2} e^{2 \pi \xi} \omega=0, \\
\frac{\partial^{2} \omega}{\partial \xi^{2}}+\frac{\partial^{2} \omega}{\partial \eta^{2}}-\frac{\operatorname{Re}}{2}\left[\frac{\partial \psi}{\partial \eta} \frac{\partial \omega}{\partial \xi}-\frac{\partial \psi}{\partial \xi} \frac{\partial \omega}{\partial \eta}\right] \\
=\frac{N \operatorname{Re}}{2}\left[\pi^{2} e^{2 \pi \xi} \omega \sin ^{2}(\pi \eta)+\sin 2(\pi \eta) \frac{\partial^{2} \psi}{\partial \xi \partial \eta}-\pi \sin 2(\pi \eta) \frac{\partial \psi}{\partial \eta}\right. \\
\left.-\cos 2(\pi \eta) \frac{\partial^{2} \psi}{\partial \xi^{2}}+\pi \cos 2(\pi \eta) \frac{\partial \psi}{\partial \xi}\right] .
\end{gathered}
$$

Equations (2.6) and (2.7) must now be solved subject to the following boundary conditions. On the surface of the cylinder, no-slip condition is applied. At far-off distances $(\xi \rightarrow \infty)$ uniform flow is imposed.

(i) On the surface of the cylinder $(\xi=0): \psi=\partial \psi / \partial \xi=0, \omega=-\left(1 / \pi^{2}\right) \partial^{2} \psi / \partial \xi^{2}$.

(ii) At large distances from the cylinder $(\xi \rightarrow \infty): \psi \sim e^{\pi \xi} \sin (\pi \eta), \omega \rightarrow 0$.

(iii) Along the axis of symmetry $(\eta=0$ and $\eta=1): \psi=0, \omega=0$.

The following pressure-Poisson equation is then solved to find the pressure in the flow field:

$$
\begin{aligned}
-\left(p_{\xi \xi}+p_{\eta \eta}\right)= & \frac{2}{r^{2} \pi^{2}}\left[\left(\psi_{\xi \eta}-\pi \psi_{\eta}\right)^{2}-\left(\psi_{\xi \xi}-\pi \psi \xi\right)\left(\psi_{\eta \eta}+\pi \psi \xi\right)\right] \\
& +\frac{N}{r^{2} \pi^{2}}\left[\frac{\sin (2 \pi \eta)}{2}\left(\psi_{\xi \xi}-\psi_{\eta \eta}-2 \pi \psi_{\xi}\right)+\cos (2 \pi \eta)\left(\psi_{\xi \eta}-\pi \psi_{\eta}\right)\right]
\end{aligned}
$$

with the following boundary conditions.

(i) On the surface of the cylinder $(\xi=0): p_{\xi}=-(2 / \mathrm{Re}) \omega_{\eta}$.

(ii) At large distances from the cylinder $(\xi \rightarrow \infty): p=1$.

(iii) Along the axis of symmetry $(\eta=0$ and $\eta=1): p_{\eta}=0$.

\section{Numerical method}

The coupled nonlinear Navier-Stokes equations are solved by applying the finite difference method and the resulting algebraic equations are solved by using the multigrid method with defect correction technique. The finite difference grid is shown in Figure 3.1, where the flow is from left to right. Here $\theta=\pi$ and $\theta=0$ form the axis of symmetry. The grid is formed by the points of intersection of circles $(\xi=$ constant $)$ and radial lines $(\theta=$ constant). The boundary conditions mentioned in the previous section are applied to the 


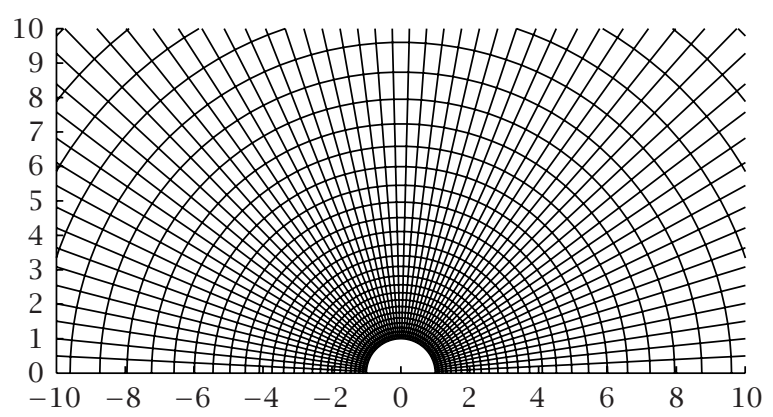

Figure 3.1. Figure showing the type of grid used.

boundaries of the grid in Figure 3.1. For this study, the finite computational domain (far field distance) is taken to be 51 times the radius of the cylinder. All derivative terms in (2.6) and (2.7) are approximated by central differences, except the convective terms in (2.7), where first-order upwind difference scheme is used to ensure diagonal dominance. This can be written as

$$
\left(F_{\theta} f_{\xi}\right)=\frac{1}{2}\left[\left(F_{\theta}+\left|F_{\theta}\right|\right)\left(f_{i, j}-f_{i-1, j}\right)+\left(F_{\theta}-\left|F_{\theta}\right|\right)\left(f_{i+1, j}-f_{i, j}\right)\right],
$$

where $f=\omega$ and $F=\psi, F_{\theta}=\partial F / \partial \theta$ and $f_{\xi}=\partial f / \partial \xi$, that is, if $F_{\theta}>0, f_{\xi}$ is approximated by backward difference and if $F_{\theta}<0, f_{\xi}$ is approximated by forward difference, and $F_{\theta}$ is approximated by central differences. In order to solve the resulting algebraic system of equations, a recursive multigrid procedure is employed in which the smoother is a point Gauss-Seidel iteration and the usual coarse grid correction is applied [9].

The initial solution is taken as $\psi=0$ and $\omega=0$ at all inner grid points except for $\psi$ at $\xi=\infty$, where the boundary condition holds. In finding the solution for higher values of Re and $N$, the solution obtained for lower values of Re and $N$ is used as starting solution. Convergence is said to have been achieved (stop condition) when the absolute difference between two successive iterations $m$ and $m+1$, at all interior grid points, is less than $10^{-7}$, that is,

$$
\epsilon=\left|\psi^{m+1}-\psi^{m}\right|<10^{-7}, \quad\left|\omega^{m+1}-\omega^{m}\right|<10^{-7} .
$$

We used the injection operator as restriction operator throughout this study. For the prolongation operator, the simplest form is derived using linear interpolation. The 9point prolongation operator defined by Wesseling [22] is used for the present study.

In order to achieve second-order accurate solution, the defect correction method is employed as follows. For example if $\mathbf{B}$ is the operator obtained by first-order upwind discretization and $\mathbf{A}$ is that obtained by second-order accurate discretization, then defect correction algorithm [9] works as given below in Algorithm 3.1. At the start of defect correction, $\bar{y}$ is a solution that is not second-order accurate, and at the end of defect correction, $\bar{y}$ is second-order accurate. 


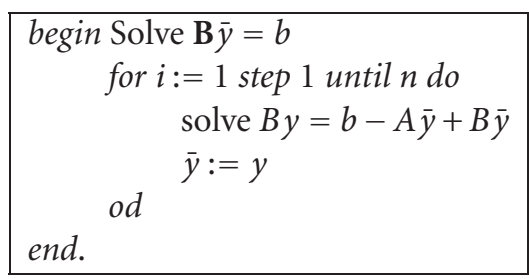

Algorithm 3.1

Usually in practice, it is sufficient to take $n=1$ or 2 . In this study we considered $n=5$ as the stop criterion for defect correction.

\section{Results and discussions}

We present the results obtained in the range of Reynolds numbers from 10-40 and for different values of interaction parameter $N$, using $512 \times 512$ as the finest grid with $256 \times$ $256,128 \times 128$ as coarser grids and $64 \times 64$ as the coarsest grid. The second-order accurate solutions converged with $\epsilon<10^{-7}$ obtained from the finest grid $512 \times 512$ are used for the discussion of the results. The drag coefficient is calculated using the following relations.

(i) Viscous drag coefficient

$$
C_{V}=-\frac{4 \pi}{\operatorname{Re}} \int_{0}^{1} \omega_{\xi=0} \sin (\pi \eta) d \eta
$$

(ii) Pressure drag coefficient

$$
C_{P}=\frac{4}{\operatorname{Re}} \int_{0}^{1}\left(\frac{\partial \omega}{\partial \xi}\right)_{\xi=0} \sin (\pi \eta) d \eta .
$$

(iii) Total drag coefficient

$$
C_{D}=C_{V}+C_{P}
$$

We first verified our results with the case of no magnetic field $(N=0)$ and found that they are in accordance with the literature values. In particular, the drag coefficient, separation length, and separation angle are in agreement with the findings of Takami and Keller [20], Dennis and Chang [5], Fornberg [6], and Lima E Silva et al. [12]. Here, we have uniformly chosen 51 times the radius of cylinder as far field distance, which is sufficiently a large domain to obtain accurate results for all values of $N$ and Re. The domains of similar size have been used in the literature for the problem of flow past a circular cylinder $[5,20]$. A comparison of the drag coefficient values is given in Table 4.1. The drag coefficient values in two different grids $256 \times 256$ and $512 \times 512$ for certain values of $N$ are presented in the Table 4.2 to show the grid independence. The effect of magnetic field on the streamlines for $\operatorname{Re}=10,20$ and 40 are presented in Figures 4.1, 4.2, and 4.3, respectively. From these figures it is evident that as the magnetic field is applied, the 
TABLE 4.1. Verification of our second-order accurate results converged to $\epsilon<10^{-7}$ with the available literature values for the case of flow with zero magnetic field.

\begin{tabular}{cccccc}
\hline Re & $\begin{array}{c}\text { Present results } \\
(512 \times 512 \text { grid })\end{array}$ & Reference [20] & Reference [5] & Reference [6] & Reference [12] \\
\hline 10 & 2.83 & 2.745 & 2.846 & 2.81 & - \\
20 & 2.02 & 2.003 & 2.045 & 2 & 2.04 \\
40 & 1.5 & 1.536 & 1.522 & 1.498 & 1.54 \\
\hline
\end{tabular}

TABLE 4.2. Comparison of second-order accurate drag coefficient values obtained in $512 \times 512$ and $256 \times 256$ grids.

\begin{tabular}{ccccccc}
\hline & \multicolumn{2}{c}{$\mathrm{Re}=10$} & \multicolumn{2}{c}{$\mathrm{Re}=20$} & \multicolumn{2}{c}{$\mathrm{Re}=40$} \\
& $256 \times 256$ & $512 \times 512$ & $256 \times 256$ & $512 \times 512$ & $256 \times 256$ & $512 \times 512$ \\
\hline 1 & 3.920 & 3.922 & 2.765 & 2.776 & 2.194 & 2.124 \\
2 & 4.837 & 4.838 & 3.419 & 3.436 & 2.59 & 2.626 \\
5 & 6.802 & 6.8 & 4.802 & 4.807 & 3.658 & 3.666 \\
\hline
\end{tabular}

recirculation bubble behind the circular cylinder is completely suppressed for $\operatorname{Re}=10$ at $N=0.4$. But with further increase of the magnetic field for $\mathrm{Re}=10$, it is found that the boundary layer separation started growing again at a critical value of $N=9$, and the boundary layer separation length and separation angle are found to increase with further increase of $N$ up to 15 . For $R e=20$ and 40 , the suppression is not complete. It is found that both the length of the recirculation bubble and separation angle decreases up to $N=2$ and increases when $N \geq 3$. The variation of the separation angle $(\theta)$ and length of the recirculation bubble $(l)$ with interaction parameter $(N)$ is depicted in Figure 4.4. It is observed that for higher values of $N$, the flow becomes straightened in the main stream direction and the curvature of the recirculation bubble (i.e., $\psi=0$ ) decreases. The flow inside the recirculation bubble slows down monotonically with increasing magnetic field. As the magnetic forces are proportional to and resist the flow of fluid in any other direction than that of the unperturbed magnetic field near the cylinder, they produce changes in the pattern of the vorticity lines. The length of the standing vortex is reduced slightly and the strength of the disturbance in front of the cylinder is increased with increasing magnetic field. These features can be seen from the figures of vorticity lines as shown in Figure 4.5 for $\mathrm{Re}=40$. It is clear from Figure 4.6 that the magnetic field tends to suppress the surface vorticity behind the cylinder.

For very low values of $N(<0.1)$, a general base pressure increase is found on both the upstream and downstream faces of the cylinder and with further increase of the magnetic field, rear pressure inversion is found, that is, the upstream base pressure increases continuously while downstream base pressure decreases. The angular evolution of the surface pressure is presented in Figure 4.7. The surface pressure at $\theta=90^{\circ}$ (the interface between upstream and downstream regions) is found to first increase with $N$ and then decrease with further increase of $N$. It is also found that the pressure at rear stagnation point varies with $\sqrt{N}$ for $N \geq 4$. This is shown in Figure 4.8. These results agree with the 
190 MHD flow around a circular cylinder

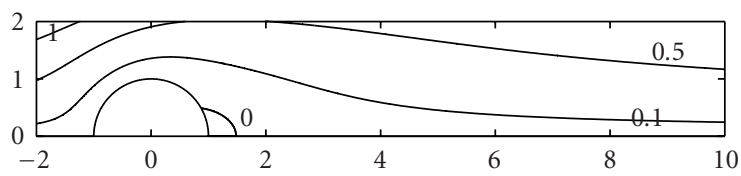

(a)

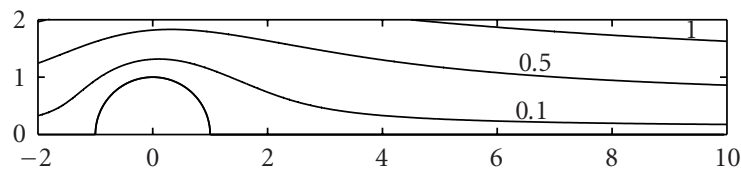

(b)

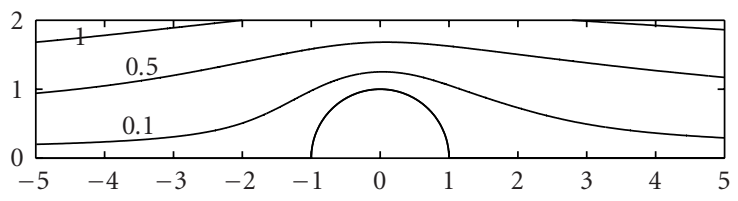

(c)

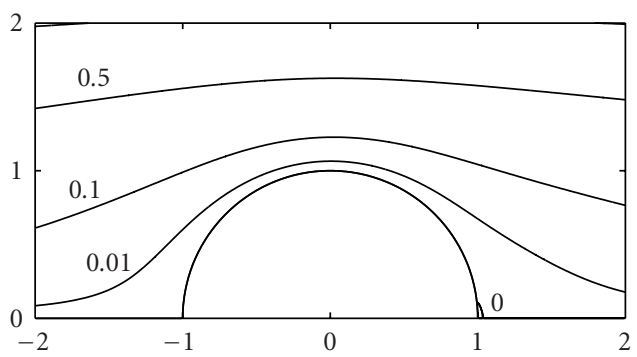

(d)

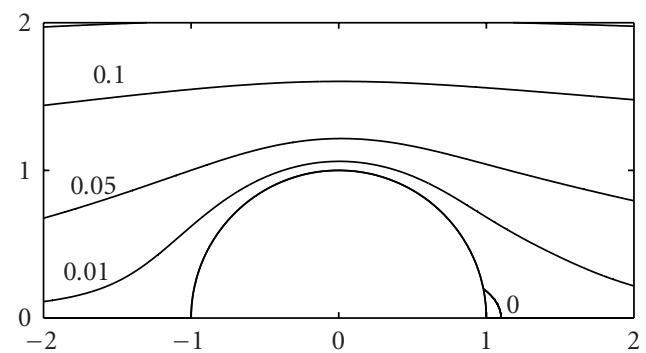

(e)

Figure 4.1. Streamlines for $\operatorname{Re}=10$. (a) $N=0$, (b) $N=1$, (c) $N=5$, (d) $N=10$, and (e) $N=15$. 
T. V. S. Sekhar et al. 191

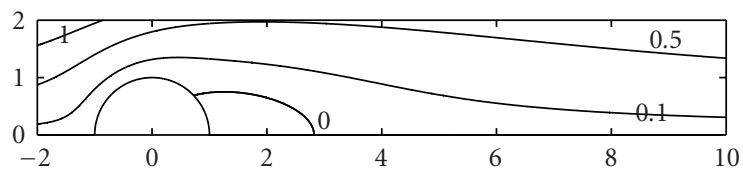

(a)

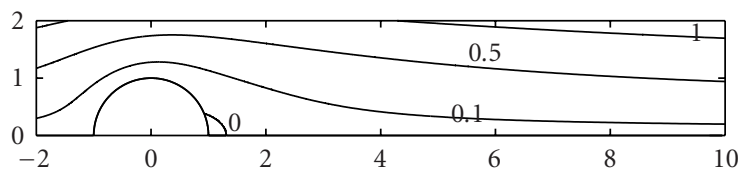

(b)

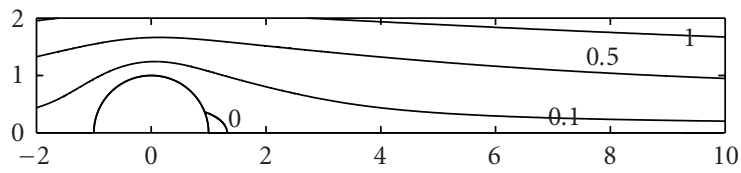

(c)

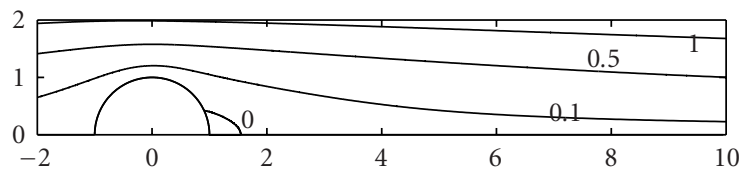

(d)

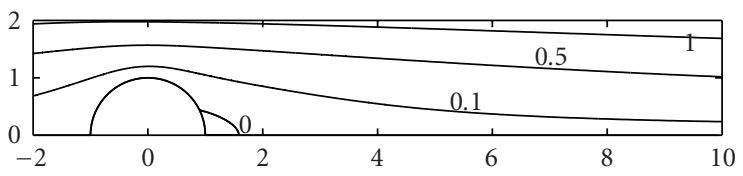

(e)

Figure 4.2. Streamlines for $\operatorname{Re}=20$. (a) $N=0$, (b) $N=1$, (c) $N=3$, (d) $N=10$, and (e) $N=12$. 
192 MHD flow around a circular cylinder

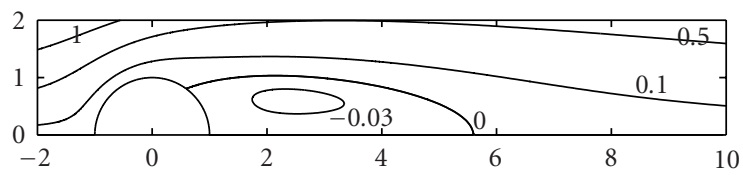

(a)

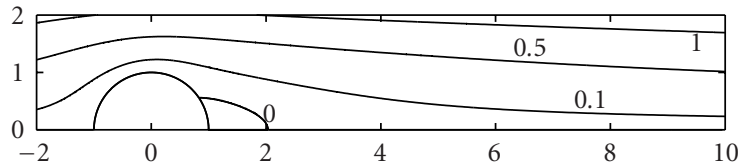

(b)

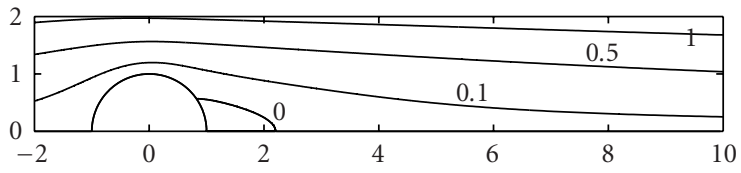

(c)

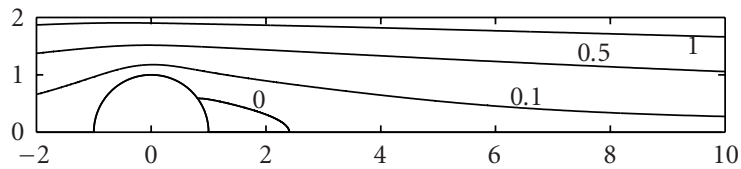

(d)

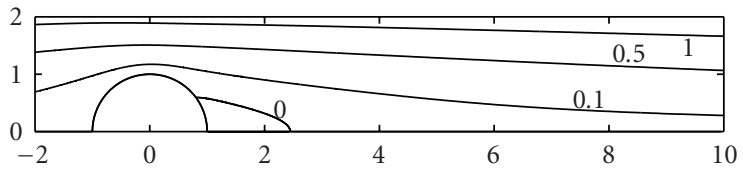

(e)

Figure 4.3. Streamlines for $\operatorname{Re}=40$. (a) $N=0$, (b) $N=2$, (c) $N=5$, (d) $N=10$, and (e) $N=12$. 
T. V. S. Sekhar et al. 193

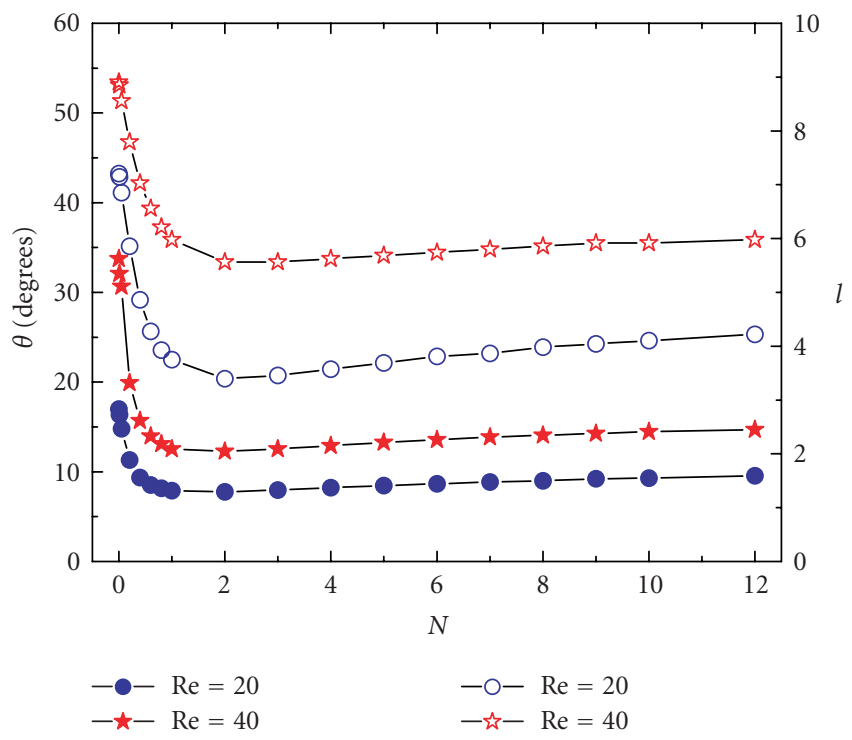

Figure 4.4. Dependence of separation angle $\theta$ (open symbols with left $y$-scale) and the separation length $l$ (closed symbols with right $y$-scale) on the interaction parameter $N$.

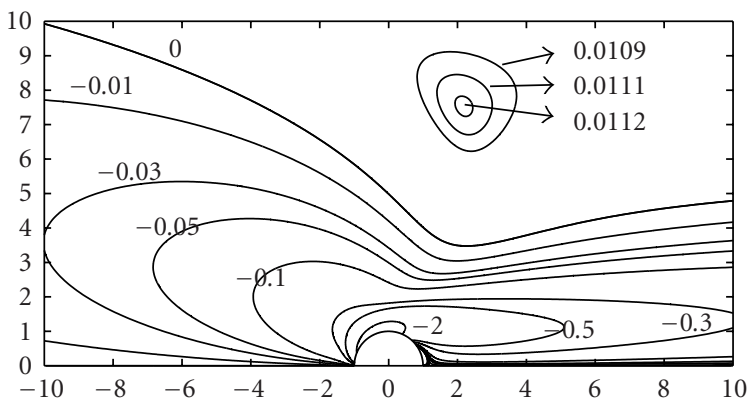

(a)

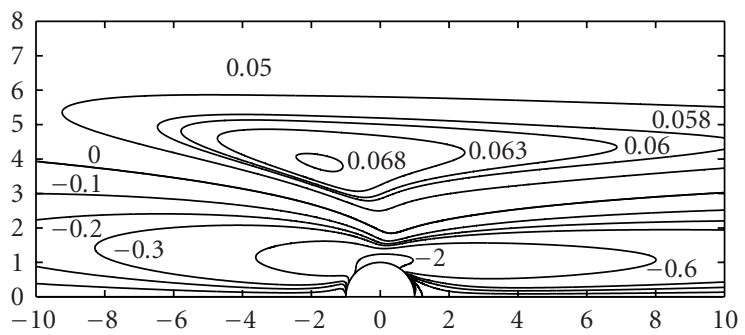

(b)

FIgure 4.5. Isocontours of vorticity for (a) $\operatorname{Re}=40, N=1$ and (b) $\operatorname{Re}=40, N=12$. 
194 MHD flow around a circular cylinder

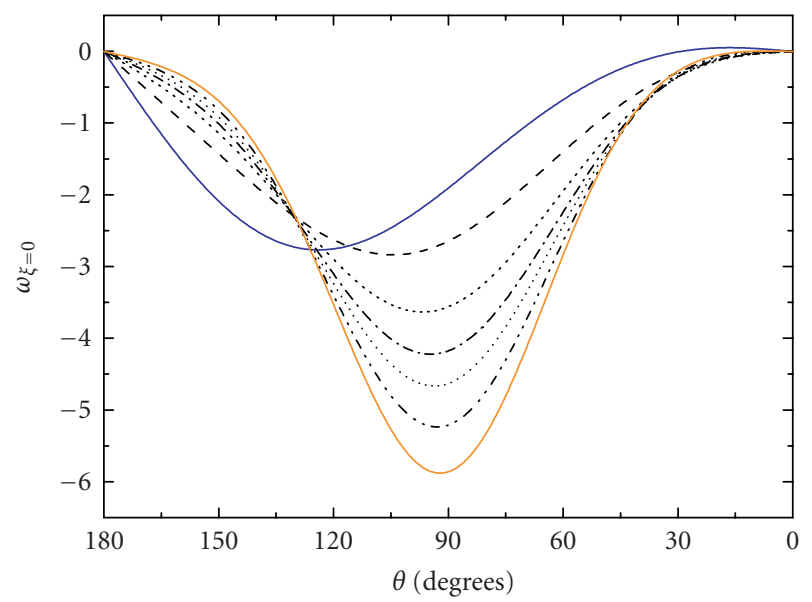
$-N=0$
$\cdots \cdots$
$\cdots \cdots$
$-\cdots-N=10$
$---N=1$
… $N=3$
$\cdots N=5$
$-N=15$

(a)

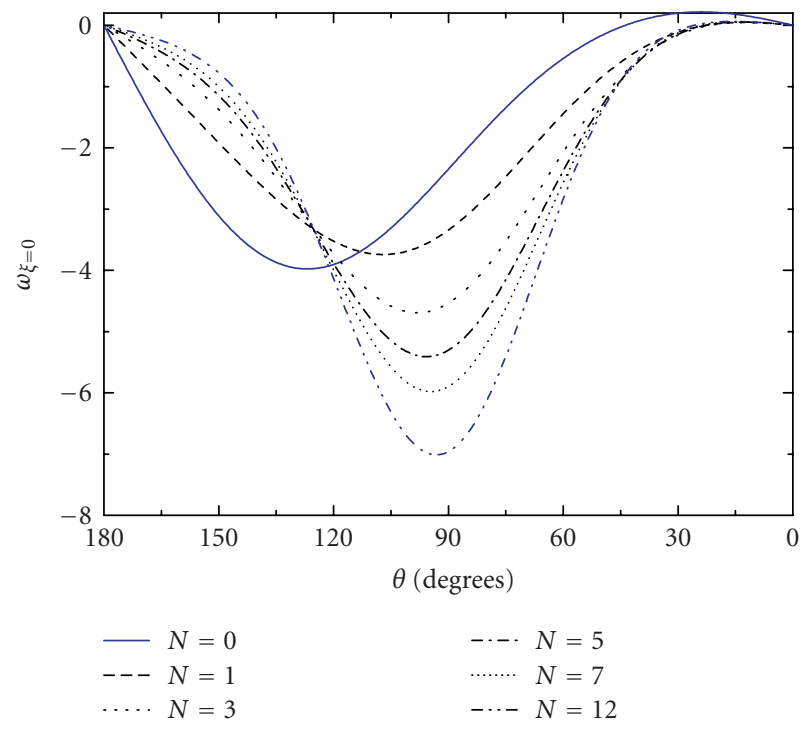

(b)

Figure 4.6. Angular evolution of surface vorticity for (a) $\operatorname{Re}=10$ and (b) $\operatorname{Re}=20$. 
T. V. S. Sekhar et al. 195

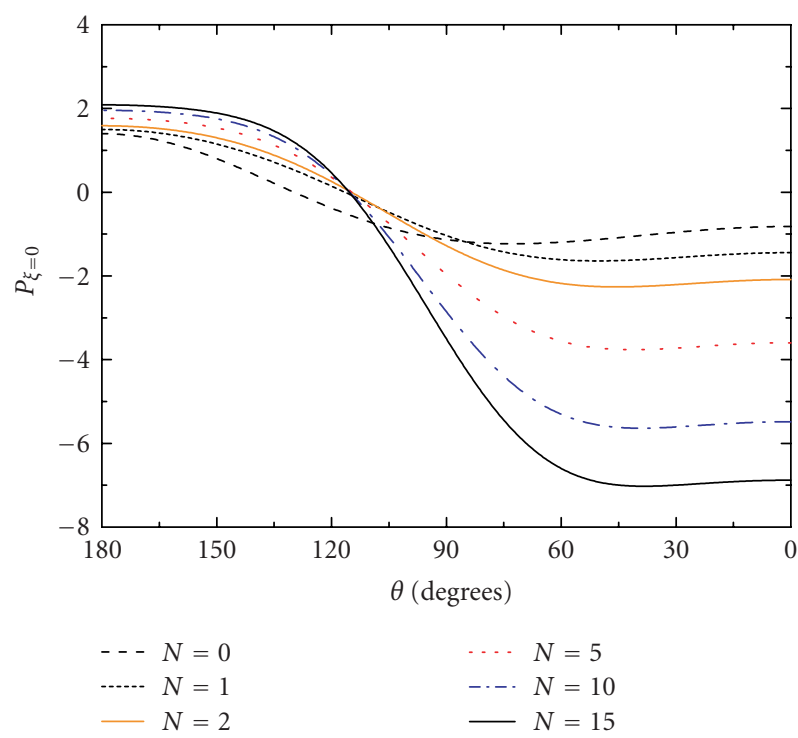

(a)

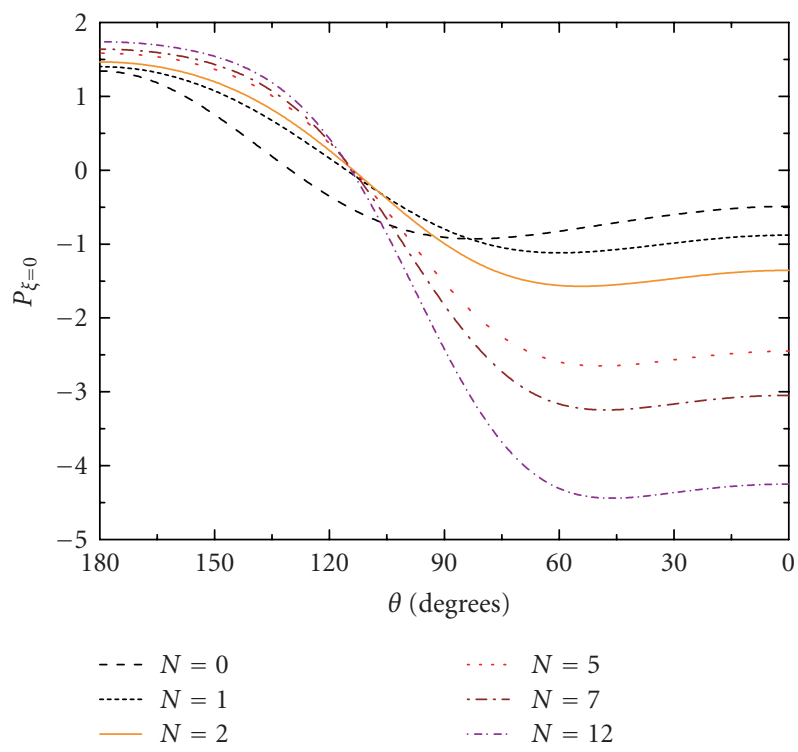

(b)

Figure 4.7. Angular evolution of surface pressure for (a) $\operatorname{Re}=10$ and (b) $\operatorname{Re}=20$.

experimental results of Josserand et al. [8]. From the plots of angular evolution of surface pressure, it is observed that in comparison with the case of no magnetic field, the 
196 MHD flow around a circular cylinder

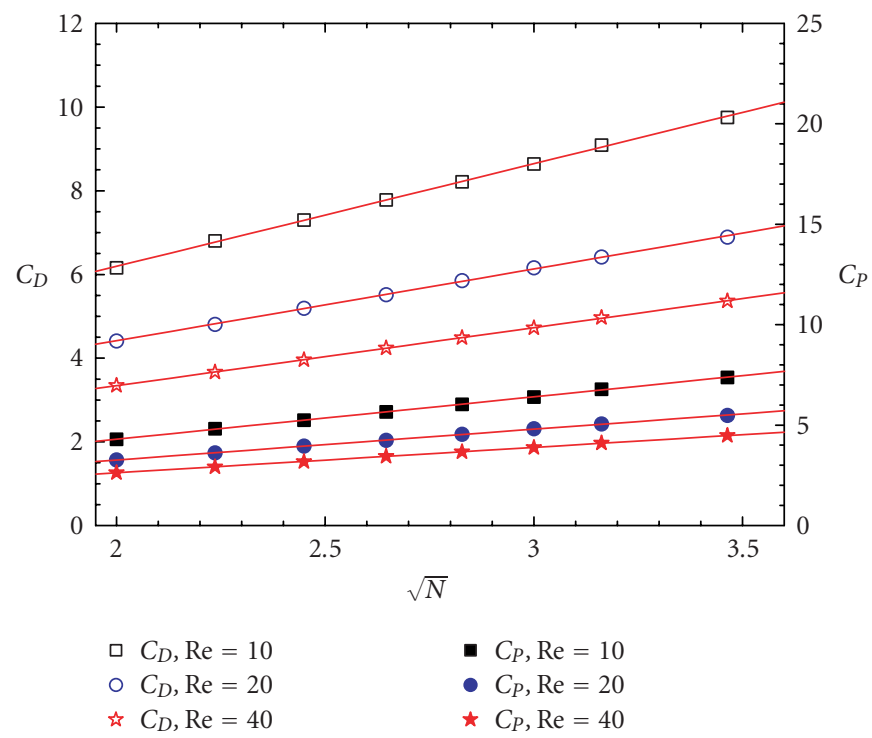

(a)

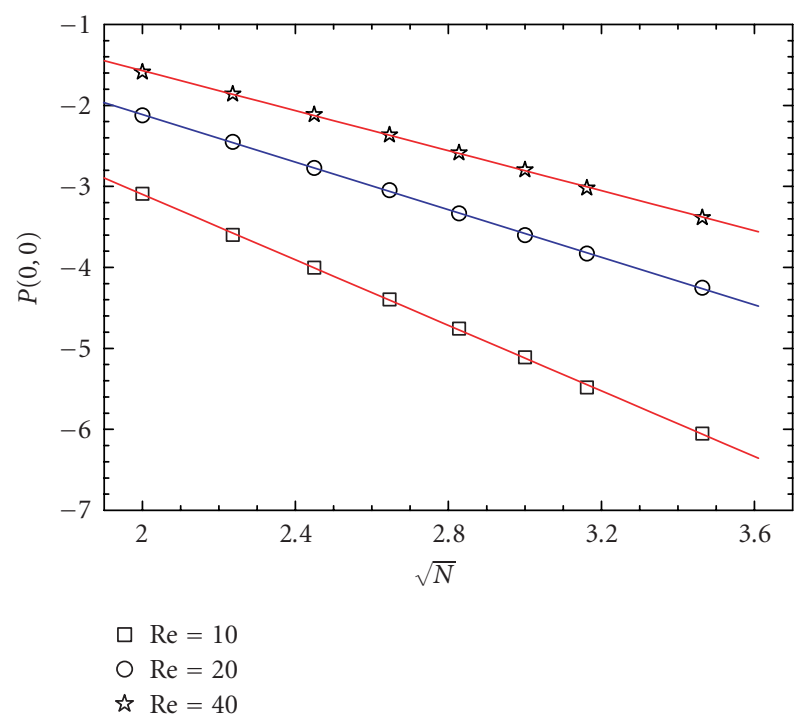

(b)

Figure 4.8. (a) Linear dependence of pressure drag coefficient $C_{P}$ and total drag coefficient $C_{D}$ and (b) the rear pressure $P(0,0)$ on $\sqrt{N}$.

increase of front pressure around the front stagnation point is in line with the hypothesis of Maxworthy [13, 14] and Josserand et al. [8] that a stagnant flow develops upstream of 


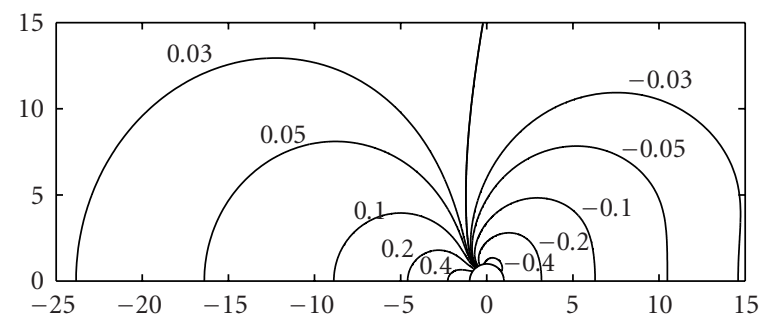

(a)

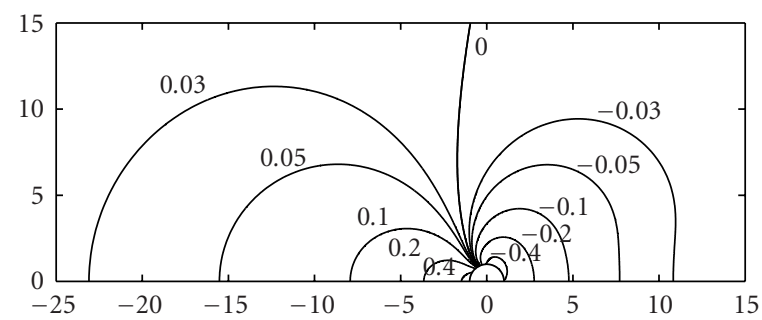

(b)

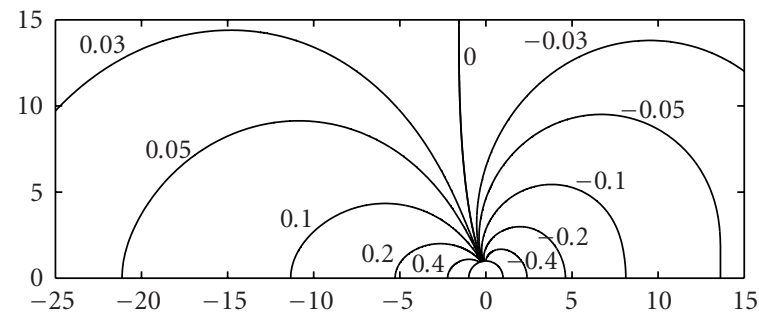

(c)

Figure 4.9. Pressure fields for the flow with $\operatorname{Re}=10$. (a) $N=0$, (b) $N=1$, and (c) $N=15$.

the sphere when the magnetic field is increased. The pressure Poisson equation (2.8) is solved and the isocontours of pressure fields around the cylinder in presence of magnetic field for $\operatorname{Re}=10,20$, and 40 are presented in Figures 4.9, 4.10, and 4.11, respectively. From these figures, it is evident that the pressure in the downstream region is reduced up to a certain value of $N$ and then increases in both upstream and downstream regions with further increase in the magnetic field. This behavior can be attributed to the nonmonotonic behavior in separation length and separation point. For the case of flow past a circular cylinder, Fornberg [7] has presented the pressure fields for $\mathrm{Re}=100-600$. The radial and transverse velocity components at $\theta=90^{\circ}$ (interface of upstream and downstream regions) are given in Figure 4.12 for $\mathrm{Re}=20$. 


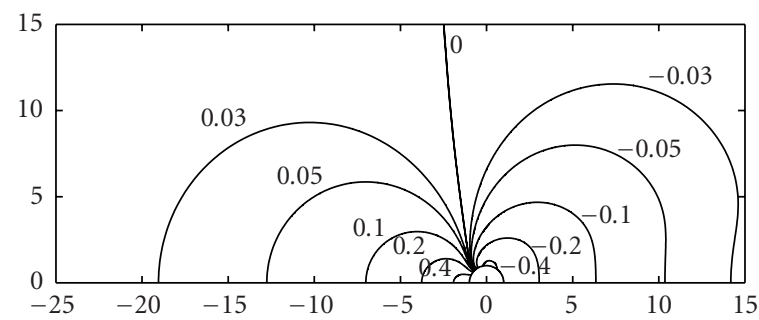

(a)

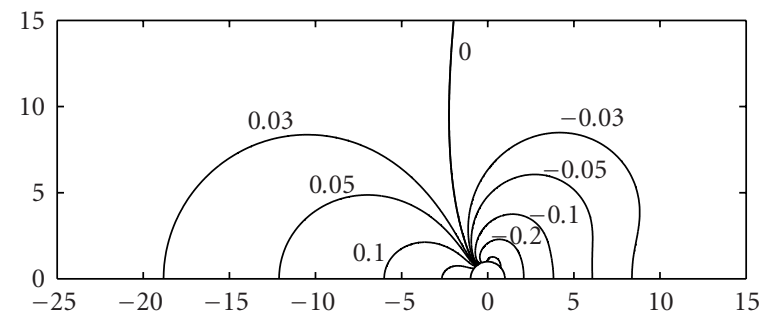

(b)

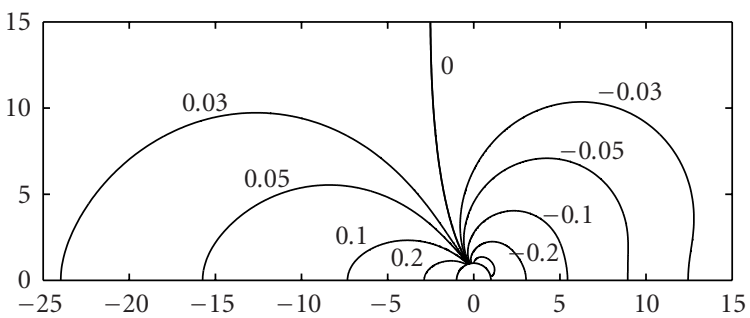

(c)

Figure 4.10. Pressure fields for the flow with $\operatorname{Re}=20$. (a) $N=0$, (b) $N=1$, and (c) $N=12$.

For very low values of $N(<0.1)$, the drag coefficient decreases when compared to the no field case. This is in agreement with Bramely $[3,4]$. The drag coefficient increases with further increase of the magnetic field. The pressure drag coefficient $\left(C_{P}\right)$ and viscous drag coefficient $\left(C_{V}\right)$ versus interaction parameter is shown in Figure 4.13. The total drag coefficient versus interaction parameter and the drag coefficient versus Re is presented in Figure 4.14. The pressure drag coefficient $C_{P}$ and the total drag coefficient $C_{D}$ are found to vary with $\sqrt{N}$ for $N \geq 4$. This behavior can be seen in Figure 4.8. The linear dependence with $\sqrt{N}$ of the pressure drag coefficient and total drag coefficient is in accordance with 


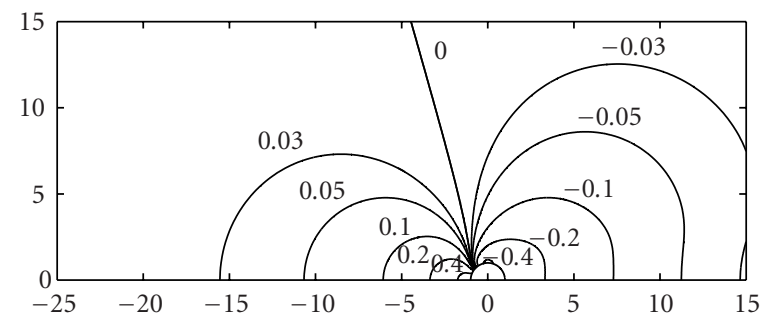

(a)

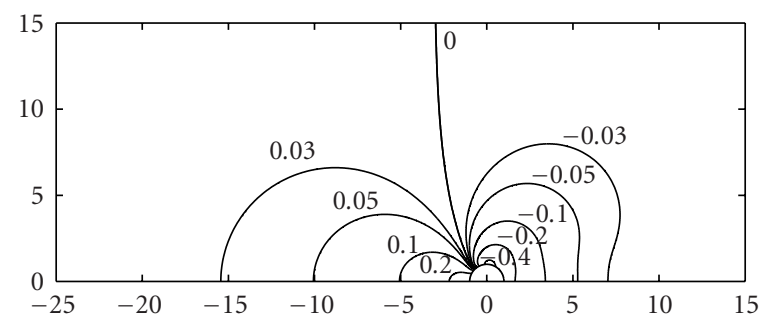

(b)

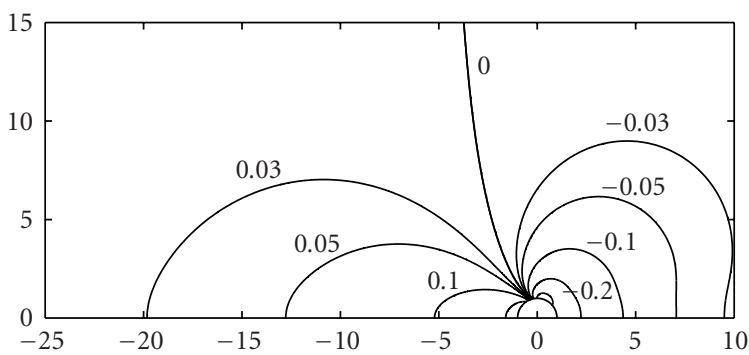

(c)

Figure 4.11. Pressure fields for the flow with $\operatorname{Re}=40$. (a) $N=0$, (b) $N=1$, and (c) $N=12$.

the experimental findings of Maxworthy [13], Yonas [23], and Josserand et al. [8]. The constant decrease of the base pressure for high $N$ is the major source of the increase in the overall drag coefficient $C_{D}$. For these values of $N$, the loss in total pressure suffered along the front streamlines under the effect of the $\mathbf{j} \times \mathbf{B}$ forces are responsible for the rear pressure drop. This behavior is responsible for the increase of pressure drag coefficient which in turn increases total drag coefficient. 


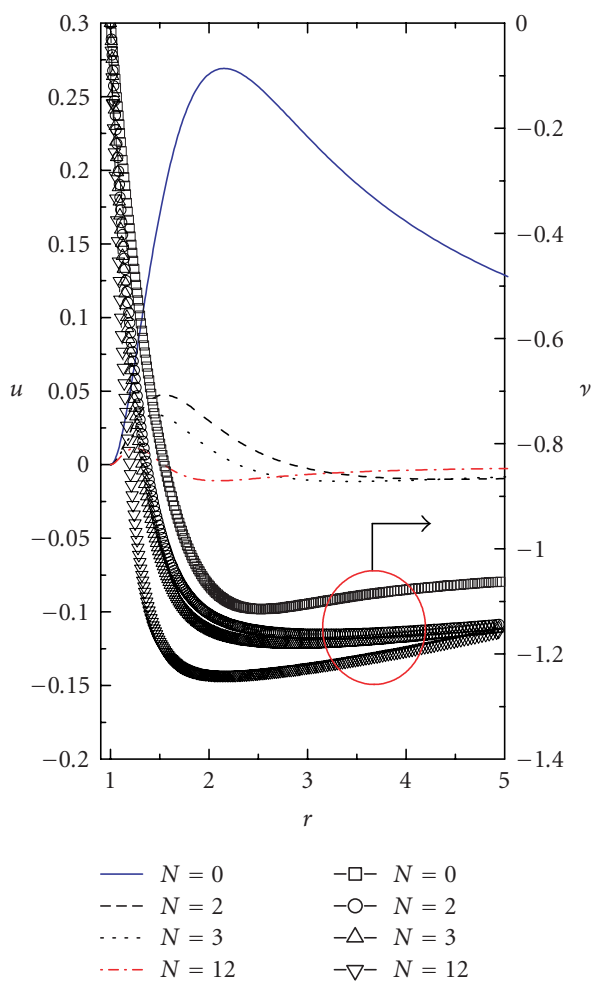

FiguRE 4.12. Variation of radial $u$ and transverse $v$ components of velocity $U$ of the flow with $\operatorname{Re}=20$. Lines without symbol represent radial velocity with left $y$-axis. The lines with symbol represent the transverse velocity with right $y$-axis.

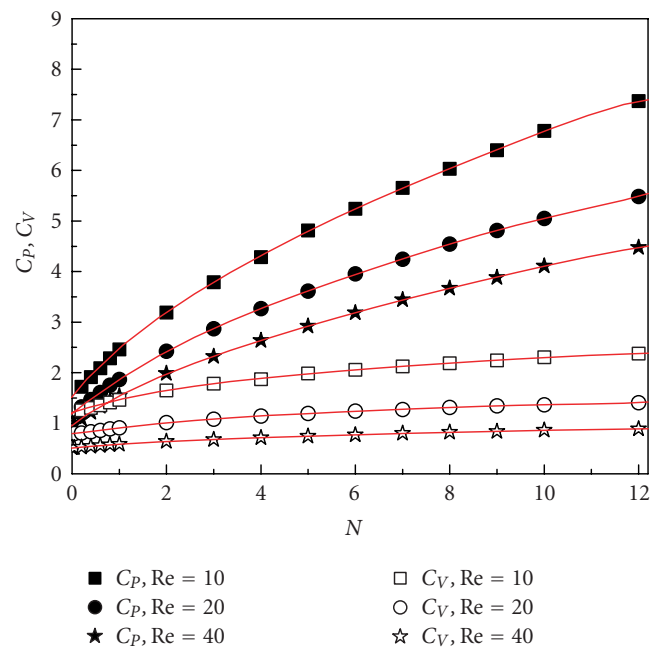

Figure 4.13. Variation of viscous drag coefficient $C_{V}$ and pressure drag coefficient $C_{P}$ as a function of interaction parameter $N$. 
T. V. S. Sekhar et al. 201

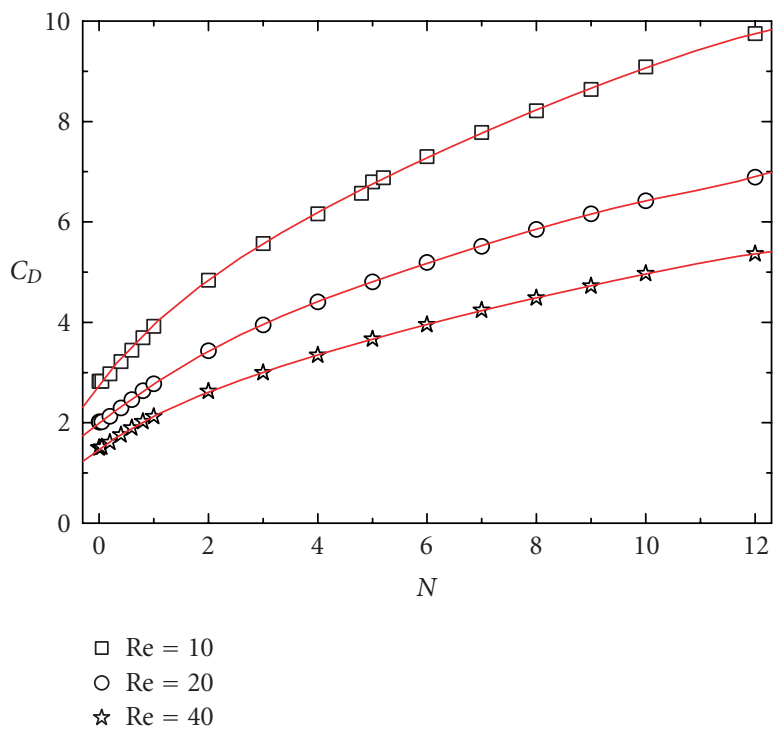

(a)

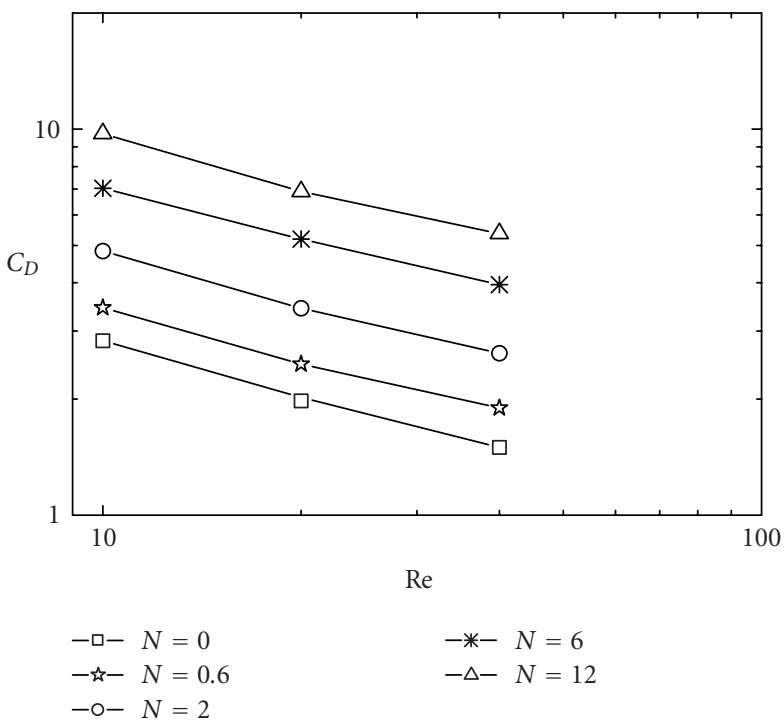

(b)

Figure 4.14. Variation of total drag coefficient $C_{D}$ as a function of (a) interaction parameter $N$ and (b) Reynolds number Re. 


\section{Conclusions and outlook}

The steady, incompressible, 2D conducting fluid flow around a circular cylinder with an applied magnetic field parallel to the main flow is investigated for moderate values of the Reynolds numbers and interaction parameters using the finite difference method. The pressure-Poisson equation is solved and pressure fields for this flow with magnetic field are presented for the first time. It is found that the boundary layer separation at rear stagnation point of the circular cylinder for $\mathrm{Re}=10$ is completely suppressed at $N=0.4$ and again starts growing when $N \geq 9$. For $\operatorname{Re}=20$ and 40, a slight increase in separation length and separation point is found when $N \geq 3$. It is also found that the pressure drag coefficient, total drag coefficient, and the pressure at rear stagnation point varies with $\sqrt{N}$. This is in agreement with experimental findings. This work can be extended for larger values of Re and $N$. The effects of the transverse magnetic field on the MHD flow around circular cylinder and sphere can also be explored. It will be interesting too, to investigate the problem when the magnetic Reynolds number is also included.

\section{Acknowledgments}

The authors are thankful to Professor Umamaheswara Rao, Department of Applied Mathematics, Andhra University, Visakhapatnam, for his encouragement. They are also thankful to Dr. Raghurama Rao, Department of Aerodynamics, Indian Institute of Science, Bangalore, for useful discussions. A part of this work was presented at the Indian Society for Theoretical and Applied Mechanics (ISTAM) conference [17].

\section{References}

[1] H. M. Bae, L. Baranyi, M. Koide, T. Takahashi, and M. Shirakashi, Suppression of Kármán vortex excitation of a circular cylinder by a second cylinder set downstream in cruciform arrangement, J. Comput. Appl. Mech. 2 (2001), 175-188.

[2] L. Baranyi, Computation of unsteady momentum and heat transfer from a fixed circular cylinder in laminar flow, J. Comput. Appl. Mech. 4 (2003), no. 1, 13-25.

[3] J. S. Bramely, Magnetohydrodynamic flow past a circular cylinder, Z. Angew. Math. Phys. 25 (1974), 409-416.

[4] - Magnetohydrodynamic flow past a circular cylinder-II, Z. Angew. Math. Phys. 26 (1975), 203-209.

[5] S. C. R. Dennis and G. Z. Chang, Numerical solutions for steady flow past a circular cylinderat Reynolds numbers up to 100, J. Fluid Mech. 42 (1970), 471-489.

[6] B. Fornberg, A numerical study of steady viscous ow past a circular cylinder, J. Fluid Mech. 98 (1980), 819-855.

[7] Steady viscous flow past a circular cylinder up to Reynolds number 600, J. Comput. Phys. 61 (1985), 297-320.

[8] J. Josserand, Marty Ph. and Alemany A. Pressure and drag measurements on a cylinder in a liquid metal flow with an aligned magnetic field, Fluid Dynam. Res. 11 (1993), 107-117.

[9] G. H. Juncu, A numerical study of steady viscous flow past a fluid sphere, Int. J. Heat and Fluid Flow 20 (1999), 414-421.

[10] J. Lahjomri, Caracterisation de la structure des sillages amont et aval d'un cylinder a petit nombre de Reynolds magnetique, thesis, Grenoble University, Grenoble, 1984. 
[11] J. Lahjomri, P. Caperan, and A. Alemany, The cylinder wake in a magnetic field aligned with the velocity, J. Fluid Mech. 253 (1993), 421-448.

[12] A. L. F. Lima E Silva, A. Silveira-Neto, and J. J. R. Damasceno, Magnetohydrodynamic flow past a circular cylinder, J. Comput. Phys. 189 (2003), 351-370.

[13] T. Maxworthy, Experimental studies in magneto-fluid dynamics: pressure distribution measurements around a sphere, J. Fluid Mech. 31 (1968), 801-814.

[14] Experimental studies in magneto-fluid dynamics: flow over a sphere with a cylindrical afterbody, J. Fluid Mech. 35 (1969), 411-416.

[15] G. Mutschke, G. Gerbeth, and V. Shatrov, Two- and three-dimensional instabilities of the cylinder wake in an aligned magnetic field, Phys. Fluids 9 (1997), 3114-3116.

[16] G. Mutschke, G. Gerbeth, V. Shatrov, and A. Tomboulides, The scenario of three-dimensional instabilities of the cylinder wake in an external magnetic field: A linear stability analysis, Phys. Fluids 13 (2001), 723-734.

[17] T. V. S. Sekhar, R. Sivakumar, and T. V. R. Ravi Kumar, MHD flow around a cylinder at moderate Reynolds numbers, presented in 49th Congress of Indian Society for Theoretical and Applied Mechanics, IIT Kharagpur (ISTAM) held at National Institute of Technology, Rourkela, 2004.

[18] Magnetohydrodynamic flow around a sphere, to appear in Fluid Dynam. Res.

[19] S. Swarup and P. C. Sinha, Magnetohydrodynamic flow past a circular cylinder, J. Appl. Math. Phys. 28 (1977), 73-83.

[20] H. Takami and H. B. Keller, Steady two-dimensional viscous flow of an incompressible fluid past a circular cylinder, Phys. Fluids 11 (1969), suppl. 51-56.

[21] K. Tamada, Flow of a slightly conducting fluid past a circular cylinder with strong aligned magnetic field, Phys. Fluids 5 (1962), no. 7, 817-823.

[22] P. Wesseling, Report NA-37, Delft University of Technology, the Netherlands, 1980.

[23] G. Yonas, Measurements of drag in a conducting fluid with an aligned magnetic field and large interaction parameter, J. Fluid Mech. 30 (1967), 813-821.

T. V. S. Sekhar: Department of Mathematics, Pondicherry Engineering College, Pondicherry 605014, India

E-mail address: sekhartvs@yahoo.co.in

R. Sivakumar: Department of Physics, Pondicherry Engineering College, Pondicherry 605014, India

E-mail address: sivtex@mailcity.com

T. V. R. Ravi Kumar: Department of Applied Mathematics, Ideal College of Arts and Sciences, Kakinada 530003, India

E-mail address: tvrravikumar@yahoo.com 


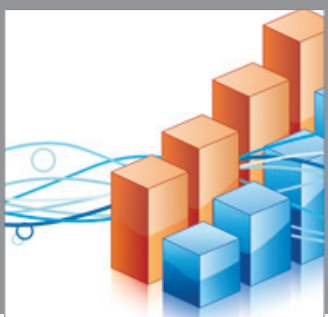

Advances in

Operations Research

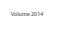

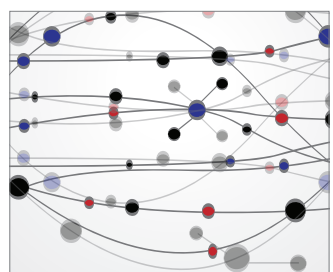

\section{The Scientific} World Journal
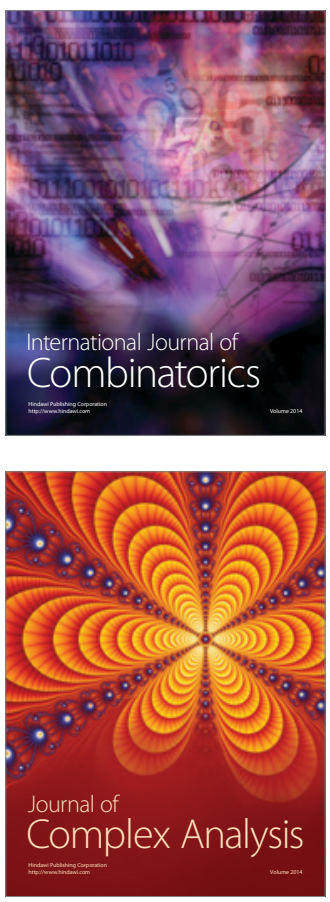

International Journal of

Mathematics and

Mathematical

Sciences
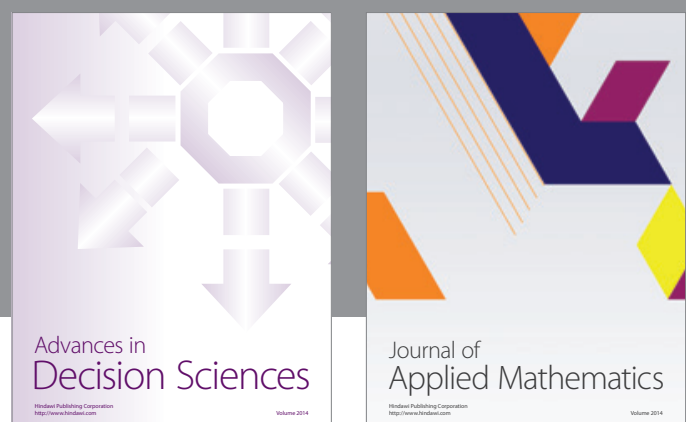

Journal of

Applied Mathematics
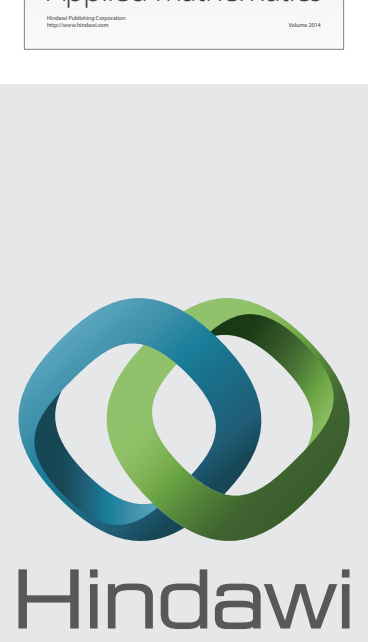

Submit your manuscripts at http://www.hindawi.com
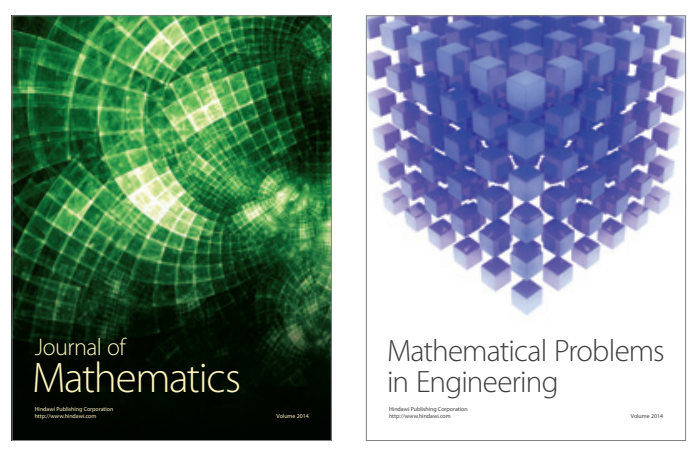

Mathematical Problems in Engineering
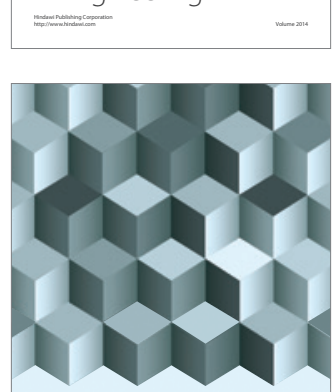

Journal of

Function Spaces
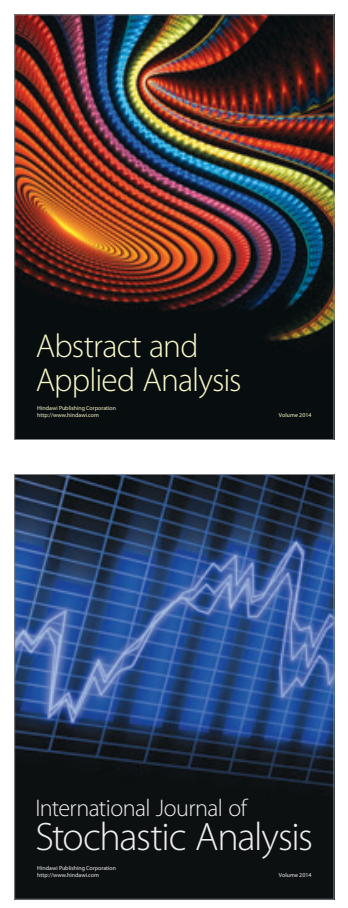

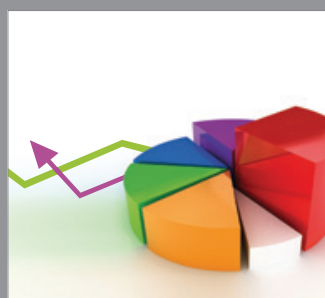

ournal of

Probability and Statistics

Promensencen
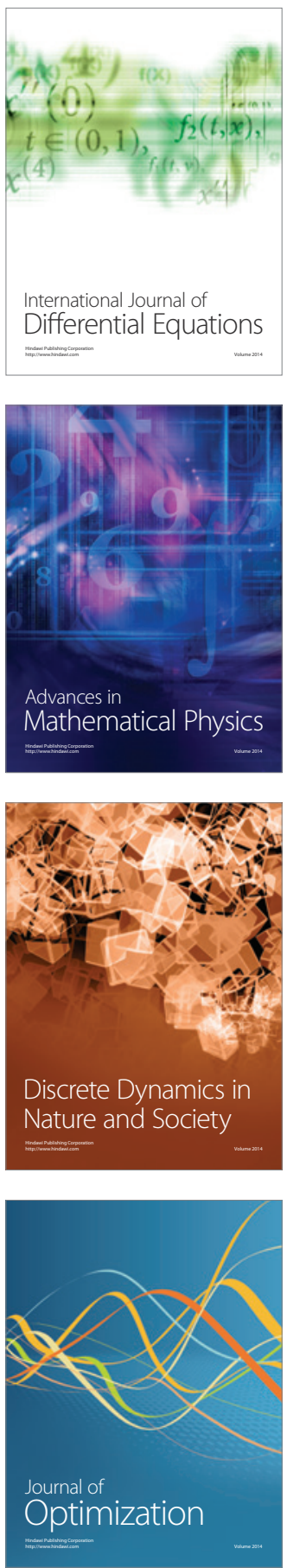